\title{
The Influence of Flower Anatomy and Apple Cultivar on the Apple Flower Phytobiome
}

\author{
Blaire Steven, Department of Environmental Sciences, Connecticut Agricultural Experiment Station, 123 \\ Huntington Street, New Haven; and Regan B. Huntley and Quan Zeng, ${ }^{\dagger}$ Department of Plant Pathology and \\ Ecology, Connecticut Agricultural Experiment Station, 123 Huntington Street, New Haven
}

Accepted for publication 20 May 2018.

\begin{tabular}{|c|c|}
\hline $\begin{array}{l}\text { The apple flower is a transient organ that can shed light on how } \\
\text { plant-associated phytobiomes are established and structured. Stigmas, } \\
\text { stamens, receptacles, and petals were dissected from flowers, and } \\
\text { the microbiome of each structure was characterized. Each flower part } \\
\text { harbored a largely overlapping set of bacterial taxa, predominantly } \\
\text { within the groups Pseudomonas and Enterobacteriaceae. } \\
\text { However, the structure of the communities differed. The stigmas } \\
\text { showed a high dominance of a small number of operational taxonomic } \\
\text { units (OTUs; } 97 \% \text { sequence identity) whereas OTUs on petals } \\
\text { were more even in relative abundance. Results from the OTU analysis } \\
\text { of phytobiomes from stigmas from three apple cultivars, Braeburn, }\end{array}$ & $\begin{array}{l}\text { Mclntosh, and Sunrise, indicated cultivar did not significantly } \\
\text { influence community structure. Correlation analysis of bacterial } \\
\text { taxa in the apple phytobiome found a negative correlation between } \\
\text { Pseudomonas and Enterobacteriaceae, suggesting a potential } \\
\text { niche exclusion relationship between these taxa. In this respect, } \\
\text { the phytobiome of the apple flower is relatively simple but different } \\
\text { flower parts, particularly the stigma, enrich the relative abundance } \\
\text { of specific bacterial populations. Correlations in the community } \\
\text { structure point to potential antagonistic relationships, which could be } \\
\text { used to manipulate the structure of the microbiome for biological } \\
\text { control of pathogens or other orchard management strategies. }\end{array}$ \\
\hline
\end{tabular}

It is increasingly recognized that the microorganisms that associate with plants, collectively referred to as the phytobiome, are important in plant development and health (Berg et al. 2014; Dicke 2016; Hawkes and Connor 2017). Yet, there are still significant knowledge gaps concerning how plants acquire their phytobiome and how those microbiomes are structured by environmental conditions or by plant-microbe interactions (Busby et al. 2017; Turner et al. 2013).

Of many different plant structures and organs, flowers represent one of the most appealing targets of research in the plant phytobiome for both their scientific interests and their importance in agriculture. Unlike many other plant organs that are present all season long, flowers are a transient structure that serves as the reproductive

${ }^{\dagger}$ Corresponding author: Q. Zeng; E-mail: quan.zeng@ct.gov

Funding: This study was supported by the Northeastern IPM Center partnership grant, USDA-National Institute of Food and Agriculture (NIFA-AFRI)-Exploratory Research grant 2016-67030-24856 to Q. Zeng; USDA-NIFA-Organic Transitions grant 2017-51106-27001, U.S. Department of Agriculture grant Hatch to Q. Zeng and B. Steven; and USDA-AFRI ELI Research/Extension Experiential Learning for Undergraduates Program (2016-06390).

*The $\boldsymbol{e}$-Xtra logo stands for "electronic extra" and indicates that two supplementary figures and two supplementary tables are published online.

(C) 2018 The American Phytopathological Society organ of the plant. The development of flowers often spans only a brief period of time, between a few days to a few weeks. This provides an opportunity to understand how the microbiome establishes, develops, and declines over the course of plant development. Furthermore, because many plants depend on other organisms for pollination they need to attract pollinators to the flower (Dudareva and Pichersky 2000). As such, specialized structures in the plant, primarily the nectary, offer a nutrient rich environment (the nectar) that act as an attractant, but also support colonization by microorganisms. Another nutrient rich environment is the stigma surface, as many hydrophilic compounds, free sugar, polysaccharides, and glycoproteins were produced on stigma pellicle to attach and hydrate pollen grains (Ferrari et al. 1981; Pusey et al. 2008). These nutrient rich environments of flower not only support large bacterial populations but also open up an opportunity for invasion by pathogens. For instance, Erwinia amylovora is the causative agent of fire blight, a devastating disease of apple and pear. It rapidly multiplies on the stigma of the flower and causes the initial infection at the nectaries on the hypanthium (Heitefuss 2012). Plant pathogens and other environmental microbes are often in an antagonistic relationship, competing for nutrients and space, thus diseases on flowers can be controlled by applying large quantity of beneficial microbes as biological controls (Stockwell et al. 2010; Sundin et al. 2016). In this aspect, understanding the microbial species composition on apple flowers, with particular focus on the stigma, will provide the knowledge 
base necessary for the development of biological controls in plant disease management.

Previous studies have documented the microbial diversity of apple flowers, but they have predominantly employed culture-based methods and were not designed to address the structure of the microbiome across flower parts or between cultivars of apples (Aleklett et al. 2014; Pusey et al. 2009; Shade et al. 2013; Vanneste 1996). In this study, we employed and optimized a culture-independent strategy to document the structure of the apple flower microbiome and how it is influenced by apple flower anatomy and apple cultivar. By comparing the bacterial community across flower parts, bacteria specific to the stigma could be identified, as they may be more likely to display antagonistic relationships with E. amylovora at the site of infection. The bacterial community inhabiting stigmas of different apple cultivars was assessed to determine if the variety of apple plays a role in shaping the microbiome. These data will inform whether biocontrol strategies can be designed for multiple apple cultivars, or if more specific targeting is required.

One of the challenges of employing culture-independent methods to describe microbial diversity of host-associated microbial communities is that the highly abundant host DNA often interferes with the amplification of the microbial DNA targets. This can be particularly daunting in plant-microbe systems because organelles of the plant, such as the chloroplast, encode their own DNA that is related to bacteria (Gray et al. 1984; Turner et al. 1999). In this regard, precautions need to be taken to ensure that amplification of host DNA is minimized in order to optimize the recovery of microbial sequences, while simultaneously ensuring that the employed methods do not introduce biases into the recovery of bacterial diversity. Peptide nucleic acids (PNAs) are RNA homologs containing a pseudopeptide backbone instead of the sugar-phosphate backbone, while retaining the geometry and the spacing of the bases to base pair with nucleic acids. In this study, PNA clamps were used to bind to apple plastid and mitochondrial sequences and physically block the PCR amplification of these sequences (Lundberg et al. 2013), and assessed for their potential to bias microbial sequence recovery. Employing these methods, we performed $16 \mathrm{~S}$ rRNA gene sequencing to characterize the bacterial communities on four flower parts, the stigma (stigma + part of style/ovary), stamen (filament + anther), petal, and receptacle. Additionally, to test if different cultivars of apple harbor different microbiomes, the stigmas of three cultivars (Braeburn, McIntosh, and Sunrise) were also characterized. Thus, these data shed light on how the apple flower phytobiome is structured across the flower, and between different apple cultivars.

\section{MATERIALS AND METHODS}

Site of apple flower collection. Flower samples were collected at the Lockwood Farm, Hamden, CT (41.406 N, 72.906 W) from Malus $\times$ domestica (apple) trees. The apple trees sampled had not been sprayed with any bactericide or antibiotics for the past 10 years. Trees of different cultivars are planted in the same section of the farm, within a radius of $0.2 \mathrm{~km}$ (Supplementary Fig. S1). The weather data prior to the day of sampling (sampling date for flower anatomy: 10 May; sampling date for apple cultivar: 5 May) are presented in Supplementary Table S1.

Dissecting apple flowers. Apple flowers were collected from a single cultivar of McIntosh on 10 May 2016. Flowers were collected from four trees, with 25 flowers from each tree. Criteria for selecting the flowers are as follows: (i) Intactness of petals (only flowers with no petal missing were selected); (ii) Opening stage of petals (only flowers with petals fully opened were selected); and (iii) color of anther (flowers with yellow, dark yellow or golden anthers were selected, and flowers with anthers turned brown were excluded). These criteria ensure flowers analyzed in this study were at the similar stage of development and had enough time for phytobiome to develop. Collected flowers were placed in sterile plastic bags, kept on ice, and brought to the laboratory within $2 \mathrm{~h}$. Each flower was dissected into four parts: stigma, stamen, petal, and receptacle (Fig. 1). For stigma samples, the pistil was cut as close as possible to the base of the stigma, so contained both the stigma and part of the pistil. We use the term stigma throughout for ease of presentation.

Each part (e.g., stigma) from the 25 flowers of one tree was placed into a sterile tube with sterile $0.5 \times$ phosphate buffered solution (PBS; $8 \mathrm{ml}$ of PBS for receptacle, $2 \mathrm{ml}$ for stigma, $3 \mathrm{ml}$ for stamen, and $20 \mathrm{ml}$ for petal). The tubes were then vortexed for $10 \mathrm{~s}$, followed by sonication for $5 \mathrm{~min}$ in a water bath sonicator, and vortexed again for another $10 \mathrm{~s}$. The PBS buffer, containing microbes washed off from each flower part, was centrifuged at 5,500 $\times g$ for $10 \mathrm{~min}$ to collect cells, and the pellet was used for DNA isolation (see below).

Apple cultivars. For describing of the association between different cultivars and the apple flower microbiome, apple flowers were collected as described above from three cultivars (Braeburn, McIntosh, and Sunrise) on 5 May 2016. For each cultivar, three trees were sampled. The stigma portion of flowers was dissected, and the stigmas from 50 flowers from one tree were placed into a sterile microcentrifuge tube. Two milliliters of sterile $0.5 \times$ PBS was added into each tube. The tubes were then vortexed for $10 \mathrm{~s}$, followed by sonication for $5 \mathrm{~min}$ in a water bath sonicator and vortexed again for another $10 \mathrm{~s}$. A total of $1.5 \mathrm{ml}$ of PBS buffer containing microbes washed off from the stigmas were centrifuged at $15,000 \mathrm{rpm}$ for $5 \mathrm{~min}$, and the pellet was used for DNA isolation.

DNA isolation and amplification of 16S rRNA genes. DNA was isolated from the microbial containing pellets described above. DNA was extracted using the DNeasy PowerSoil Kit (Qiagen) following manufacturer's instructions. The amount of template DNA added in the PCR reaction $(50 \mu \mathrm{l})$ ranged between 8.5 to $17.6 \mathrm{ng}$. The 16S rRNA gene fragments were amplified using the $16 \mathrm{~S} \_515 \mathrm{~F}$ and $16 \mathrm{~S} \_806 \mathrm{R}$ primers with Illumina sequencing adaptors (16S_515F: TCG TCG GCA GCG TCA GAT GTG TAT AAG AGA CAG GTG CCA GCM GCC GCG GTA A; 16S_806R: GTC TCG TGG GCT CGG AGA TGT GTA TAA GAG ACA GGG ACT ACH VGG GTW TCT AAT, at final concentration of $0.25 \mu \mathrm{M}$ ), which amplify the V4 region of the 16S rRNA gene and have been employed previously to characterize plant-associated microbes (Elmer et al. 2017). PCR amplification consisted of $95^{\circ} \mathrm{C}$ for $45 \mathrm{~s}$, followed by 38 cycles of $95^{\circ} \mathrm{C}$ for $15 \mathrm{~s}, 78^{\circ} \mathrm{C}$ for $10 \mathrm{~s}, 60^{\circ} \mathrm{C}$ for $30 \mathrm{~s}$, and $72^{\circ} \mathrm{C}$ for $30 \mathrm{~s}$. For the PNA clamps treated samples, mPNA and pPNA clamps (mPNA: GGC AAG TGT TCT TCG GA and pPNA: GGC TCA ACC CTG GAC AG [Lundberg et al. 2013]) were added to the PCR reaction at a final concentration of $0.75 \mu \mathrm{M}$.

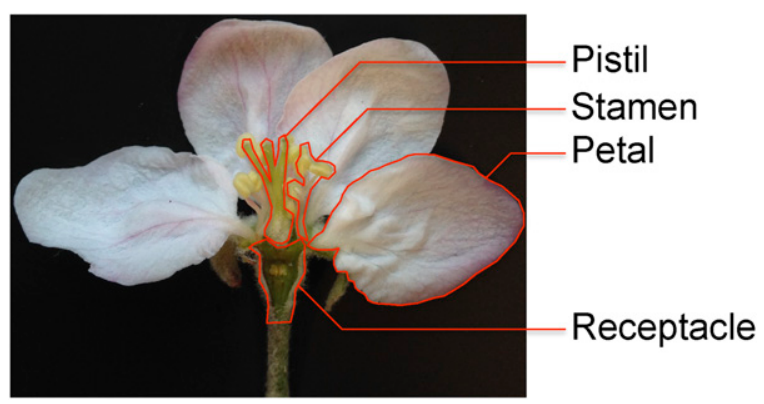

Fig. 1. Illustration of the apple flower anatomy used for phytobiome analysis in this study. 
PNA clamps were heated to $65^{\circ} \mathrm{C}$ to dissolve all precipitates prior to adding to the PCR reaction. The PNA clamps were synthesized by Panagene Inc (Daejeon, South Korea).

PCR products were analyzed by electrophoresis in $1.5 \%$ (wt/vol) agarose gels, which confirmed amplification product size being $\sim 300 \mathrm{bp}$. Amplification products were purified using the PromoKine PCR Purification Kit (PromoCell GmbH). The purified DNA was used as input to prepare Illumina sequencing library using the Nextera XT Index Kit (Illumina Inc.) following the manufacturer's instructions. The first and second PCR clean up steps were performed by using the PromoKine PCR Purification Kit. The libraries were normalized to the final concentration of $10 \mathrm{ng} \mu \mathrm{l}^{-1}$ before pooling and sequencing. The prepared libraries were sequenced on the Illumina MiSeq system at the Yale Center for Genome Analysis using standard $2 \times 150$ base pair chemistry.

The sequence datasets are available in the NCBI SRA repository (https://www.ncbi.nlm.nih.gov/bioproject/412245).

Sequence processing. Demultiplexed sequences were assembled into contigs and quality screened in the mothur software package (version 1.39.5) (Schloss et al. 2009). All sequences were selected to be at least $255 \mathrm{bp}$ in length, contain no ambiguous bases, and no homopolymers of more than $8 \mathrm{bp}$. Chimeric sequences were identified with the mothur implementation of VSEARCH (Rognes et al. 2016), and all potentially chimeric sequences were removed. The resulting sequence counts per sample are presented in Supplementary Table S2.

Sequences were clustered into operational taxonomic units (OTUs) using a $97 \%$ sequence identity threshold, employing the OptiClust algorithm in mothur (Westcott and Schloss 2017). Taxonomic classification of sequences was performed with the Naïve Bayesian classifier (Wang et al. 2007) against the SILVA reference alignment (release 128) (Quast et al. 2013) in the mothur software package.
Classification of sequences was only reported for sequences with bootstrap probabilities $\geq 80 \%$ confidence.

Statistical analyses. Prior to descriptive statistical analyses, datasets were randomly subsampled to the size of the smallest dataset (omitting outliers), resulting in 29,812 sequences per dataset. Heatmaps of taxonomic bins were generated in the STAMP software package (Parks et al. 2014). Clustering of samples was performed with the average neighbor unweighted pair group method with arithmetic mean (UPGMA). Descriptive diversity statistics were calculated in mothur. Alpha diversity was determined with the nonparametric Shannon's diversity index (H') (Spellerberg and Fedor 2003), evenness with Shannon's evenness index (Hill et al. 2003), and the estimated coverage with Good's coverage (Good 1953). Statistically significant differences in diversity statistics were identified with a one-way analysis of variance with posthoc Tukey's honestly significant difference using the agricolae R package (de Mendiburu 2017; R Core Team 2013).

Significant differences in OTU relative abundance were tested for with the ALDEx2 package. Prior to identifying significant differences, OTU count data were transformed using the centered log-ratio and normalized through Monte Carlo sampling with Bayesian sampling of 128 Dirichlet instances (Fernandes et al. 2014). Both the Kruskal-Wallis and generalized linear model tests were performed and an OTU was considered to be significantly different in relative abundance if the $P$ value was $\leq 0.05$ after $P$ values were adjusted for multiple testing with the BenjaminiHochberg correction.

The ternary plot of OTU relative abundance was generated with the ggtern extension package to $\mathrm{R}$ (Hamilton 2016). Correlations between taxonomic bins were determined using Spearman's rank correlation coefficient and the resulting correlation matrix was visualized with the corrplot R package (Wei and Simko 2017).
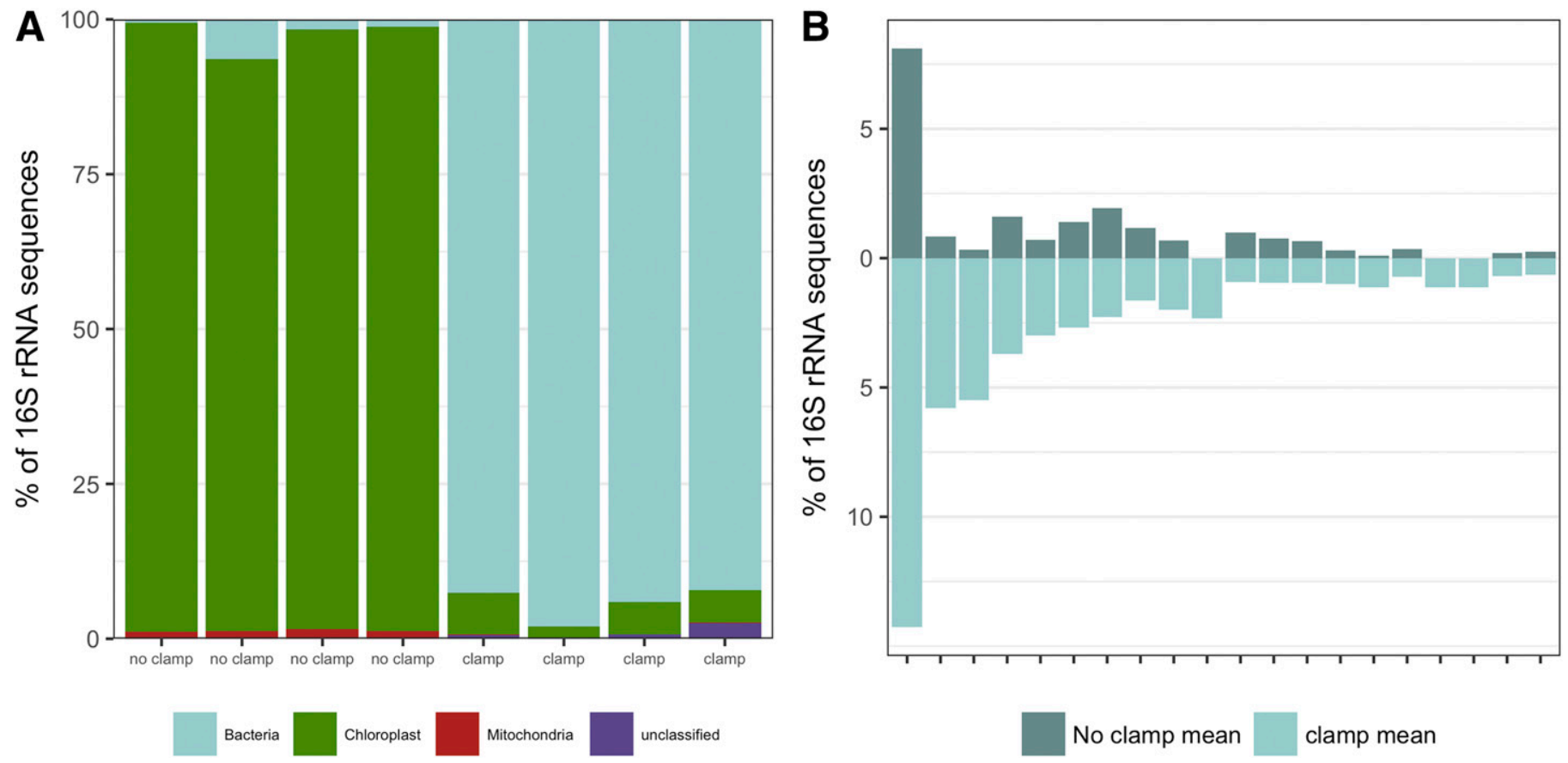

Fig. 2. Effects of including peptide nucleic acid (PNA) clamps on bacterial sequence recovery and community composition. A, Each bar represents a sample amplified with no clamps or the same sample amplified with PNA clamps. Samples are in the same order such that the first no clamp sample is the same sample as the first clamp sample. B, Each bar represents a bacterial operational taxonomic unit (OTU) identified in the dataset. The OTUs displayed represent the 20 most abundant OTUs in the dataset. The average relative abundance of each OTU in the no-clamp and PNA clamp samples is indicated by the color of the bar. 


\section{RESULTS}

Effects of including PNA Clamps in PCR. Bacterial sequence recovery. $\mathrm{PCR}$ amplification of $16 \mathrm{~S}$ rRNA genes was performed without and with PNA clamps to assess the recovery of bacterial sequences. The proportion of bacterial sequences recovered without clamps ranged from a low of $0.6 \%$ to a high of $6.4 \%$ (Fig. 2A). In comparison, including the PNA clamps in the PCR amplification consistently increased bacterial sequence recovery to greater than $92 \%$. The majority of the nonbacterial sequences were from chloroplasts with a small contribution from mitochondria (Fig. 2A). These data indicate that the inclusion of PNA clamps greatly reduce amplification of host DNA derived from plant organelles and thereby increase the recovery of bacterial sequences.

Community composition. The potential of the PNA clamps to introduce biases into the composition of the bacterial sequences recovered was also characterized. Sequences from the datasets were binned into OTUs (97\% sequence identity) and the presence and abundance of the OTUs was assessed in the PNA clamp and noclamp amplified samples. Significantly more bacterial OTUs were identified in the PNA clamp samples (mean 5,714) compared with the no-clamp samples (mean 319), reflecting the increased recovery of bacterial sequences with the inclusion of the clamps. The most abundant OTUs were present in both clamp and no-clamp amplified datasets (Fig. 2B), suggesting that addition of the PNA clamps did not bias the recovery of bacterial sequences.

Flower anatomy and the apple flower phytobiome. Taxonomic composition. At the phylum level, 32 bacterial phyla were represented among the sequences, of which the ten most abundant are displayed in Figure 3A. Across the flower parts, sequences were predominantly identified as Proteobacteria, which consistently accounted for $>90 \%$ of sequences (Fig. 3A). Cluster analysis of the datasets at the phylum level did not identify any grouping of the flower parts, suggesting flower anatomy was not a strong driver of

\section{A Phylum level taxonomic bins}

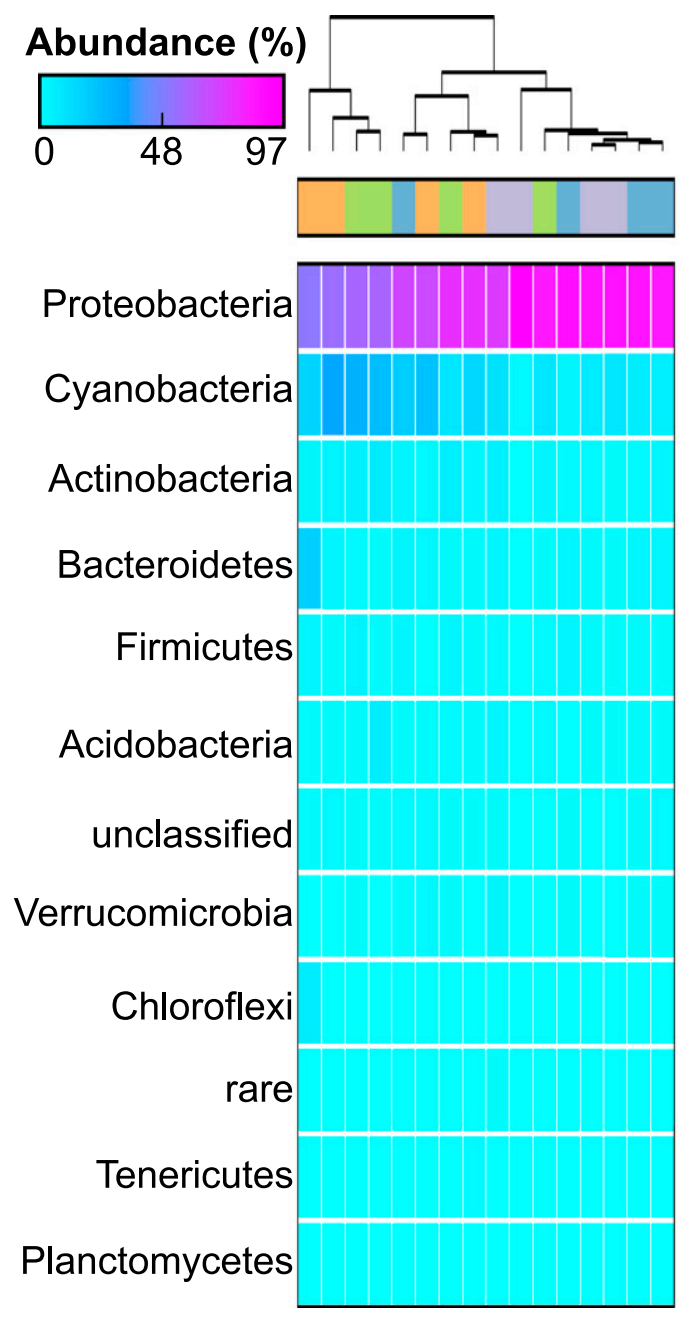

B Proteobacteria taxonomic bins

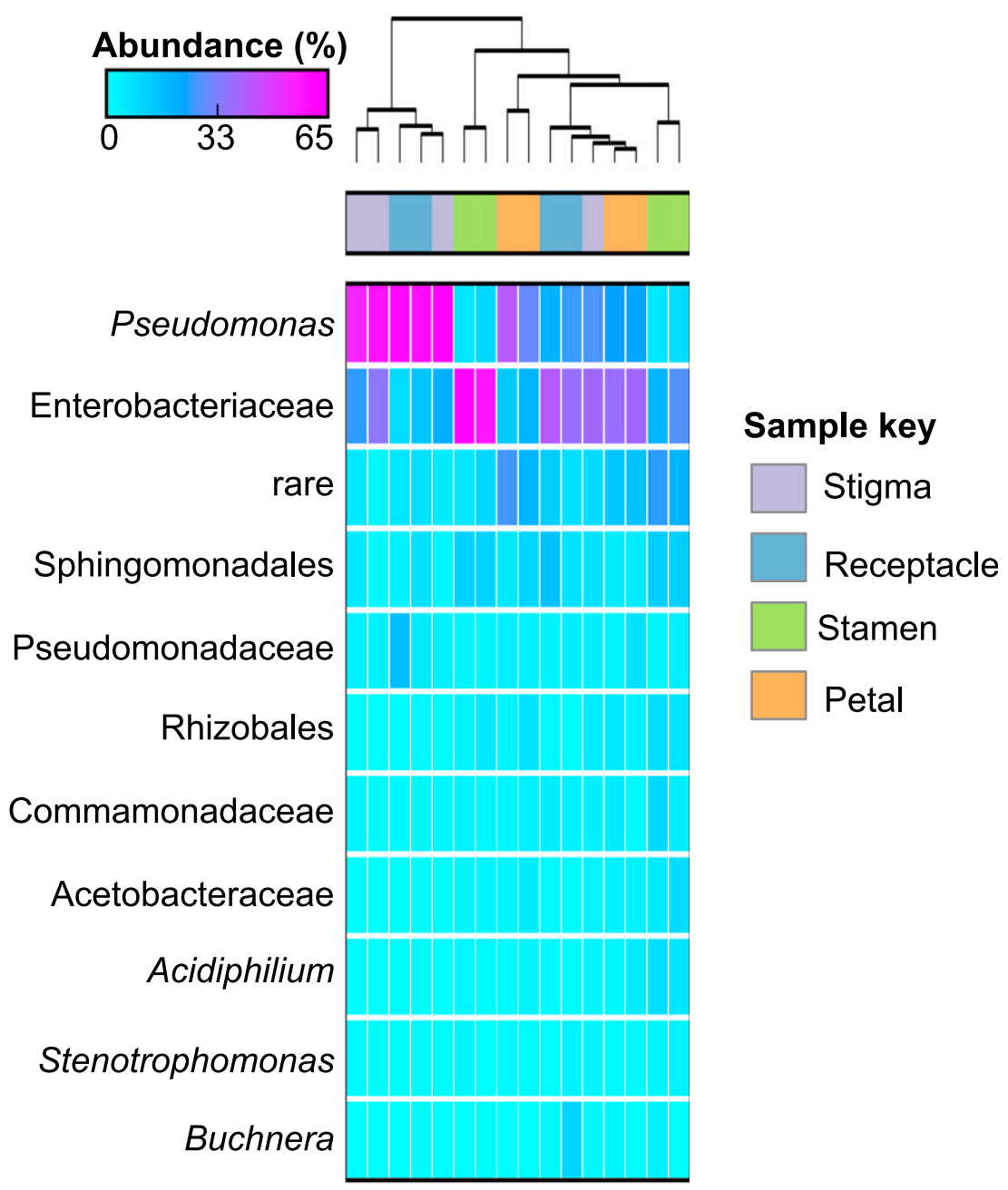

Fig. 3. Taxonomic composition of bacterial communities associated with different flower parts. A, Phylum level taxonomic bins. The 10 most abundant phyla are displayed along with sequences that were unclassified. The category "rare" represents the total of the remaining phyla. Rows are ordered by the mean abundance of each category in the dataset. Each column represents a single sample and the colored bars at the top of the display indicate the identity of the sample. Columns are ordered based on average neighbor clustering with the resulting dendrogram also displayed. B, Taxonomic bins within the phylum Proteobacteria. Each category represents the deepest level to which sequences could be reliably classified. The category "rare" represents the total of the remaining taxa. Rows are ordered by the mean abundance of each category in the dataset. Each column represents a single sample and the colored bars at the top of the display indicate the identity of the sample. Columns are ordered based on average neighbor clustering with the resulting dendrogram also displayed. All flower parts were from Mclntosh apples. 
microbiome composition at this level. Given that Proteobacteria dominated datasets, sequences within this phylum were classified to deeper taxonomic ranks. Sequences classified to the genus Pseudomonas and the family Enterobacteriaceae were the most prevalent taxonomic assignments (Fig. 3B).

Richness and diversity. The phytobiome of different apple parts was characterized to assess how flower anatomy interacts with microbial diversity. As differences in sequencing effort have been found to affect diversity estimates (Gihring et al. 2012), each dataset was randomly subsampled to the size of the smallest dataset $(29,812$ sequences). This subsampling resulted in the elimination of one petal and one stigma dataset, as they were significantly

TABLE 1

Richness and diversity of the phytobiome on different flower parts

\begin{tabular}{lcccc}
\hline Flower part & OTUs $^{\mathrm{y}}$ & $\mathrm{H}^{\prime}$ & Evenness & Coverage \\
\hline Receptacle & $1,023 \mathrm{a}^{\mathrm{z}}$ & 3.3 & 0.46 & $98.0 \mathrm{a}$ \\
\hline Petal & $1,274 \mathrm{ab}$ & 3.9 & 0.53 & $97.4 \mathrm{~b}$ \\
\hline Stamen & $790 \mathrm{bc}$ & 3.7 & 0.55 & $98.5 \mathrm{bc}$ \\
\hline Stigma & $434 \mathrm{c}$ & 2.4 & 0.39 & $99.3 \mathrm{c}$
\end{tabular}

y Operational taxonomic units (OTUs) represent sequence bins at $97 \%$ sequence identity, from sequence pooled rarified to the same sampling depth $(29,812$ sequences).

z Samples labeled with different letters were identified as significantly different (analysis of variance difference of means with posthoc Tukey's test). under-sampled in comparison with the other datasets. The richness and diversity of the sequence datasets are presented in Table 1. The number of OTUs recovered was significantly lower in the stigma compared with the other flower parts. In comparison, the highest OTU richness was recovered from the petals. The OTU recovery was mirrored in the alpha diversity where the mean $\mathrm{H}^{\prime}$ was lowest in the stigmas. Evenness, a measure of the homogeneity in the distribution of diversity, was also lowest in the stigmas indicating populations with both highly abundant and rare types (Table 1). Across the datasets, the estimated coverage was greater than $97 \%$, indicating that the sampling effort achieved in this study was sufficient to recover the majority of the bacterial diversity expected to occur in these samples.

Conservation of OTUs. As shown in Table 1, the number of OTUs recovered from the distinct flower parts was significantly different. This indicates that not all OTUs were shared between the flower parts. To investigate the conservation of OTUs among flower parts, every OTU that was present at a relative abundance of at least $1 \%$ of any sequence library were characterized. This filtering identified 18 OTUs sufficiently abundant, of which 15 were conserved between all four of the flower parts (Fig. 4). While the majority of the OTUs were conserved between the flower parts, their relative abundance often varied over a large range. For example, an OTU within the genus Pseudomonas accounted for an average of $\sim 40 \%$ of stigma sequences compared with $<10 \%$ in the stamens. The evenness of the datasets is also visually displayed. The stigma samples clearly demonstrate a high dominance of a single OTU within the genus Pseudomonas whereas petals show a more similar abundance in the dominant OTUs (Fig. 4).
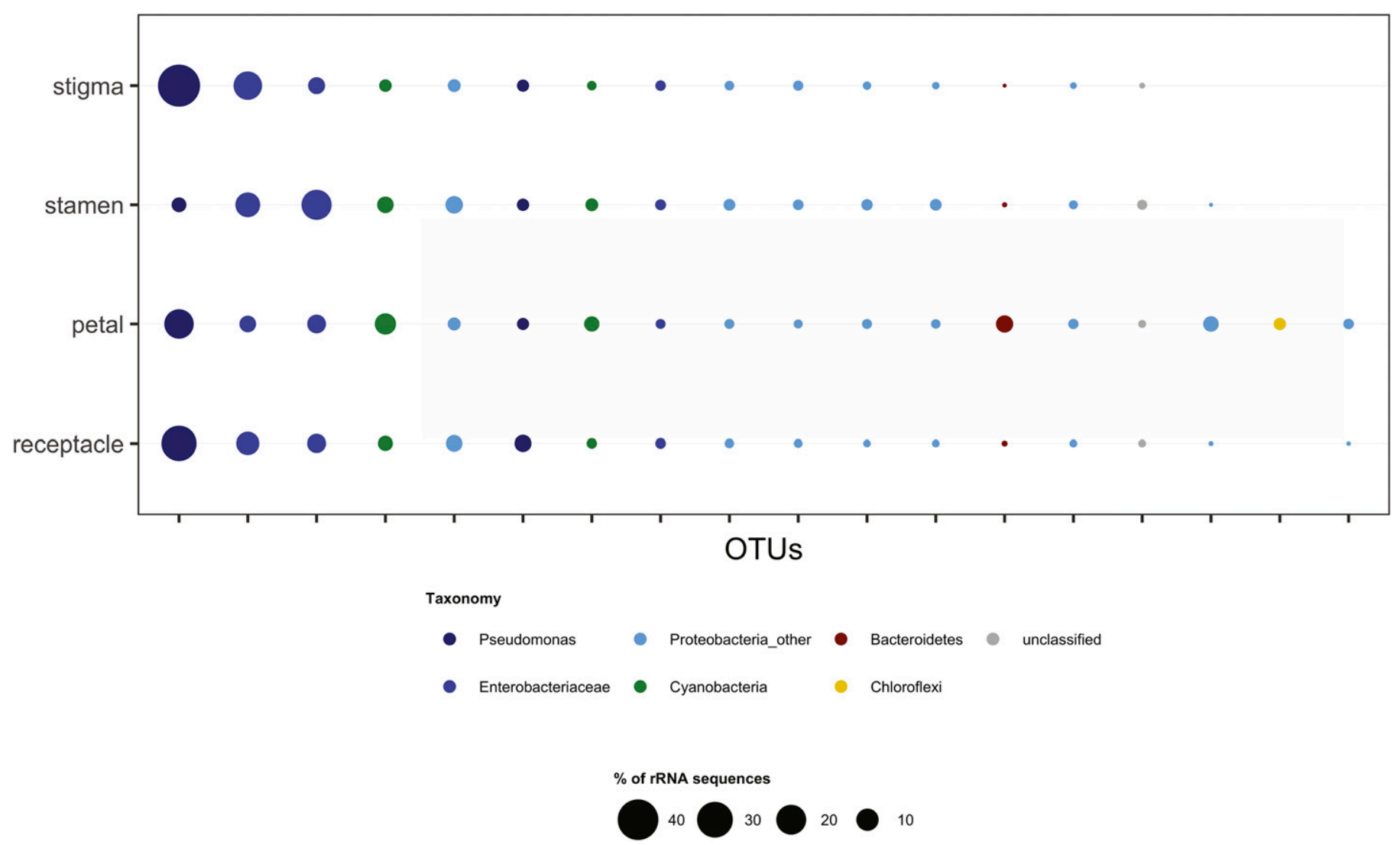

Fig. 4. Operational taxonomic unit (OTU) conservation among flower parts. Each column represents an OTU that was made up of at least $1 \%$ of sequences in any of the sequence libraries (18 total). The size of each point is representative of the mean relative abundance of the OTU in each flower part. Points are colored by the taxonomic bin to which the OTU belongs. The category "Proteobacteria other" encompasses all sequences within the Proteobacteria not belonging to Pseudomonas or Enterobacteriaceae. All flower parts were from McIntosh apples. 
Despite the variation in relative abundance of OTUs between the flower parts, no OTUs were identified where the difference was found to be statistically significant. This is in part due to the large variation of relative abundance between samples, suggesting that the membership of the flower microbiome is relatively stable, whereas the structure of the community may be much more variable (Supplementary Fig. S2).

Apple cultivars and the stigma phytobiome. Stigmas were collected from three apple cultivars to compare the composition of stigma-associated bacterial communities among apple cultivars. As previously described for the different flower parts (Fig. 3B) the stigma bacterial communities were predominantly composed of sequences within the Pseudomonas and Enterobacteriaceae (Fig. 5A). Datasets did not show any pattern of clustering, indicating apple cultivar was not a substantial driver of stigma microbiome composition. Similarly, at the OTU level the majority of OTUs were shared among all three of the apple cultivars (Fig. 5B). Finally, OTU relative abundance data were used to test for significant differences between apple cultivars. No OTUs were identified that were significantly different between any of the three cultivars. Collectively, these observations indicate that in general, apple cultivars raised in the same orchard share a common set of bacterial community members, at least among the stigmas.

Correlations in bacterial community structure. Observationally, one pattern that emerged in the community composition of the datasets was that samples with large proportions of Pseudomonas tended to have lower populations of Enterobacteriaceae, and vice versa (Figs. $2 \mathrm{~B}$ and $4 \mathrm{~A}$ ). To test the interrelationship between taxonomic bins, a correlation matrix was calculated for the different stigma datasets (Fig. 6). As suggested by the descriptive community profiles, there was a negative correlation between Pseudomonas and bacteria in the family Enterobacteriaceae, indicating that elevated abundances of one of these taxa is associated with a decrease in the other (Fig. 6). In addition, the Enterobacteriaceae were negatively correlated with most of the other dominant taxonomic bins, compared with Pseudomonas that was positively correlated with groups such as the Pseudomonadaceae, Massilia, and Actinomycetospora (Fig. 6). Collectively, these data suggest that there may be specific interactions among microbiome populations that drive the structure of the overall community.

\section{A Taxonomic bins}

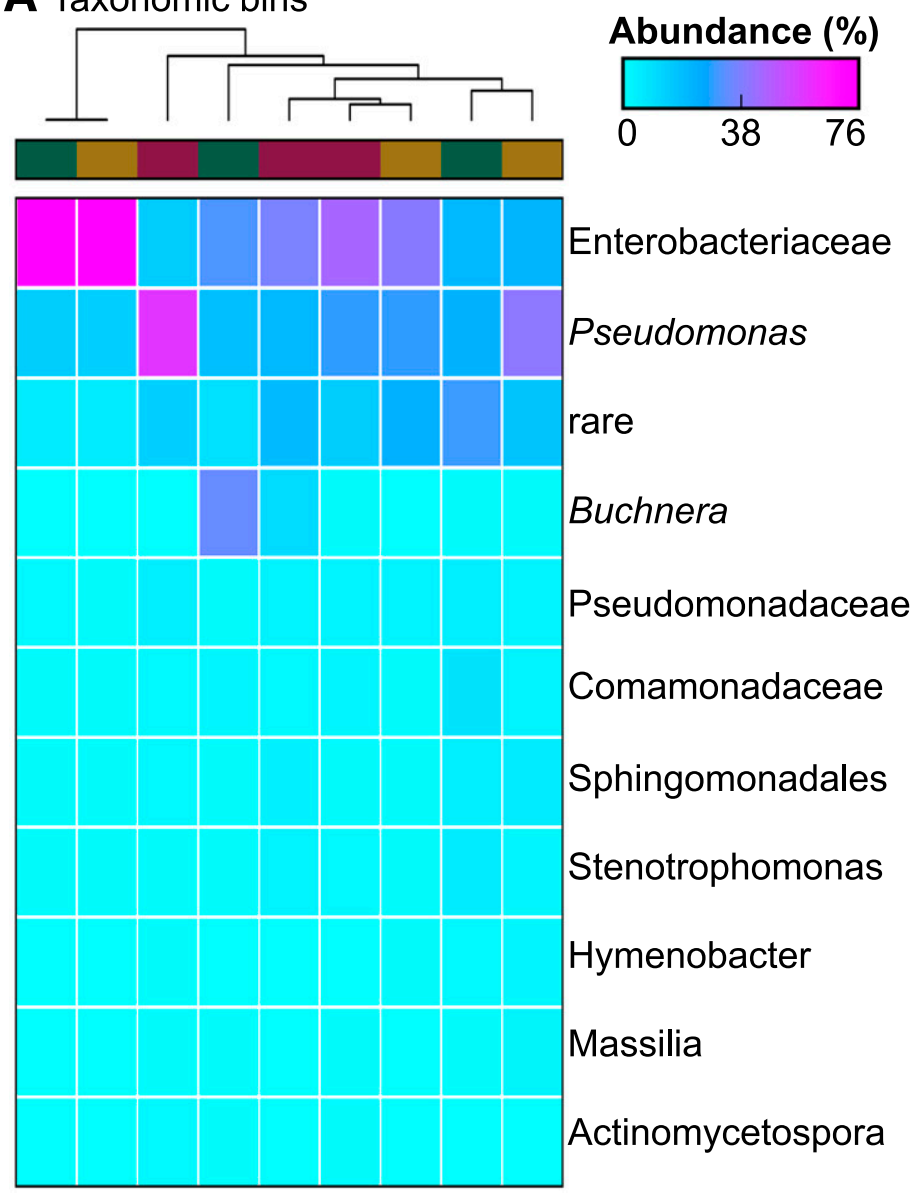

\section{B OTU abundance}

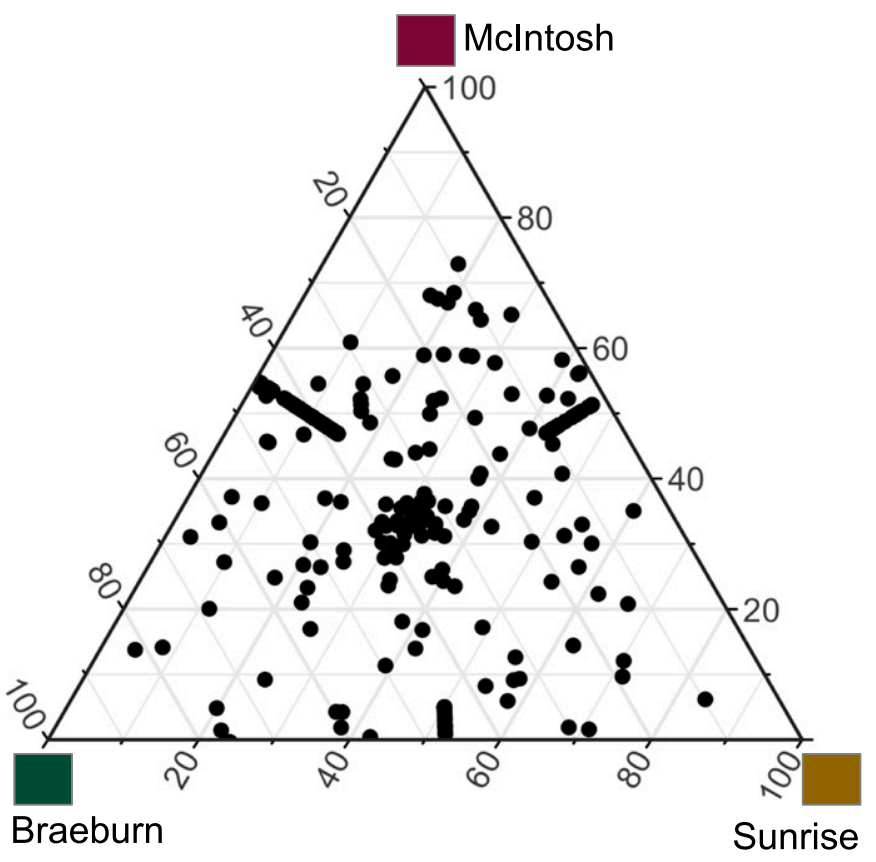

\section{Sample key} Braeburn

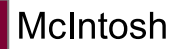

\section{Sunrise}

Fig. 5. A, Taxonomic composition of phytobiomes of different apple cultivars. Each category represents the deepest level to which sequences could be reliably classified. The category "rare" represents the total of the remaining taxa. Rows are ordered by the mean abundance of each category in the dataset. Each column represents a single sample and the colored bars at the top of the display indicate the identity of the sample. Columns are ordered based on average neighbor clustering with the resulting dendrogram also displayed. B, Ternary diagram displaying the average relative abundance of operational taxonomic units (OTUs) among the three apple cultivars. Each point represents an OTU made up of at least three sequences, as this is the smallest number that could be shared between all cultivars. 


\section{DISCUSSION}

In the absence of the PNA clamps, $>90 \%$ of the recovered sequences were derived from apple cell organelles, predominately chloroplasts (Fig. 2A). Addition of the PNA clamps greatly increased microbial sequence recovery, and appeared to have a minimal effect of the bacterial OTUs recovered (Fig. 2B). This matches observations for other plant-microbe systems, such as agave (Coleman-Derr et al. 2016), maize (Johnston-Monje et al. 2016), and sugarcane (de Souza et al. 2016) and adds to a growing body of literature that supports this method as a mechanism to characterize the phytobiome.

A previous study employing primers specifically designed to avoid amplification of chloroplast DNA, in contrast to PNA clamps, found apple flowers in Wisconsin dominated by bacterial taxa TM7 and Deinococcus-Thermus (Shade et al. 2013). This is in contrast to the results presented here, in which a large majority of the bacterial diversity belonged to the phylum Proteobacteria, particularly the genus Pseudomonas and family Enterobacteriaciae (Figs. 3A and $5 \mathrm{~A}$ ). The results of this study are more congruous with culturebased studies (Pusey et al. 2009; Stockwell et al. 1999), and fatty acid analysis (Johnson and Stockwell 1998), which also tend to find dominant populations of Pseudomonas. In this respect, either apple flowers in Wisconsin have a dramatically different composition of the microbiome compared with apples from this and previous studies, or the selection of PCR methods has a large effect on the recovered microbial diversity. These results highlight the potential difficulties in comparing studies employing different methods to characterize microbial diversity.

The data presented here show apple flower microbiomes dominated by a relatively small number of taxa and a limited influence of flower anatomy on the composition of the microbiome. Overall, the

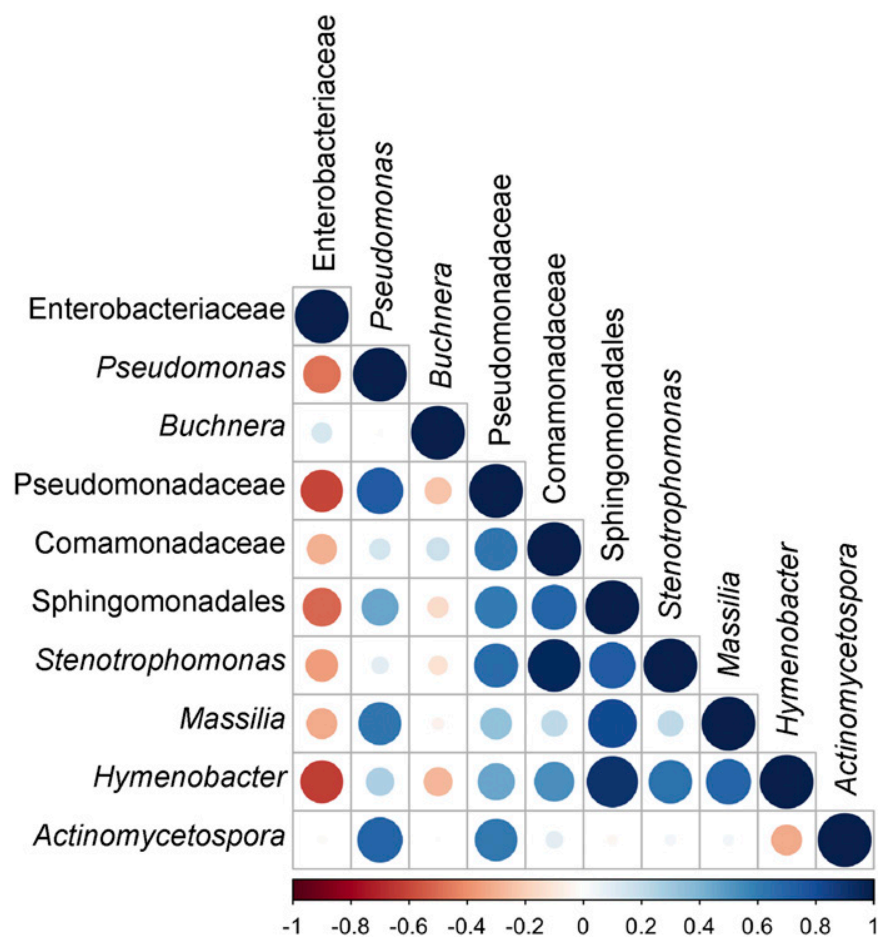

Fig. 6. Spearman's rank correlation matrix of the dominant bacterial taxa across apple stigma samples. The ten most abundant taxa are shown. Strong correlations are indicated by large circles, whereas weak correlations are indicated by smaller circles. The colors of the scale bar denote the nature of the correlation with 1 indicating perfect positive correlation (dark blue) and -1 indicating perfect negative correlation (dark red) between two taxa. stigma, stamen, petal, and receptacle of the flower shared a common set of OTUs (Fig. 4). This is not surprising, as the development of apple flowers occurs within a relatively short period of time (from the "silver tip" to "full bloom" is about 50 days, and from "tight cluster" to "full bloom" is about 15 days), and the microbiome on all flower parts likely originates from epiphytic and endophytic microorganisms near the site of flower emergence. On the other hand, certain variations in the structure of the community were observed in different flower parts, potentially associated with their respective microenvironment. For example, stigmas supported a both less diverse and even community compared with other flower parts (Table 1, Fig. 4). This was associated with a large increase in the proportion of the community made up by the most dominant OTU, which was within the genus Pseudomonas (Fig. 3). This could be due to the fact that Pseudomonas may be better adapted to the sugary, moist environment of apple stigmas and displayed a more vigorous growth pattern than many other microorganisms. In addition, Pseudomonads are prodigious producers of antimicrobial compounds, which may suppress the growth of other microorganisms (Turner et al. 2013), thus resulting in a less diverse and even community structure. In contrast, the petals, the largest sun-exposed structures of the flower, supported larger proportions bacterial taxa capable of photosynthesis, namely Cyanobacteria and Chloroflexi. Cyanobacteria have been found as epiphytes in higher plants and may contribute to plant health through biological nitrogen fixation (Fürnkranz et al. 2008). Taken together, these data suggest that while different flower parts harbor a largely overlapping set of bacterial taxa, the structure of the community may point to the function these communities play in plant health and development.

Despite flower anatomy or apple cultivar not being associated with a strong structuring of the flower microbiome (Figs. 3 and 5), patterns in the assembly of the microbiome among taxa were examined. These associations were tested by a correlation analysis. The Enterobacteriaceae were negatively correlated with all of the other dominant taxa, indicating that samples that maintain large populations of Enterobacteriaceae tend to have lower populations of other taxa (Fig. 6). In contrast, the genus Pseudomonas was positively associated with other Pseudomonadaceae, Actinomycetospora, and Massilia (Fig. 6). These interactions may support previously described relationships. For example, an examination of the bacteria found on the surface of different fruits and vegetables found significantly different bacterial community structures among produce, primarily driven by alterations in the relative abundance of Enterobacteriaceae (Leff and Fierer 2013). These data support that the Enterobacteriaceae are a common member of the fruit and vegetable microbiome.

It is important to note here that the fire blight pathogen E. amylovora is a member of the family Enterobacteriaceae (Martinec and Kocur 1964). Much of the research into apple flower microbial ecology has been in the search for bacteria antagonistic to this organism. Previous studies have documented a potential role for the organism Pseudomonas fluorescens as a biocontrol agent of fire blight (Wilson and Lindow 1993). The authors proposed that the method of action of this organism in halting E. amylovora infection is competitive exclusion. The correlational data presented here lends support to this hypothesis. However, several caveats to the correlational data presented here need to be addressed. First, sequencing data are compositional in nature. As taxa abundances from sequencing are proportional abundances, an increase in one taxon necessitates the recovery of fewer of the remaining taxa, leading to a negative correlation bias (Gloor and Reid 2016). Thus, an increase in Pseudomonas cells on the stigma, with no corresponding change in the Enterobacteriaceae biomass, will present as an increase in Pseudomonas and a decrease in Enterobacteriaceae, which could be misleading in regard to the actual relationship. 
In summary, our data suggests that flower anatomy selects for different bacterial communities but apple cultivar was not a primary driver of the stigma phytobiome composition. However, several sources of variation that were not assessed could account for not detecting specific apple blossom-bacterial relationships. For example, even though efforts were taken to select flowers with the similar physical appearance during sample collection (e.g., color of stamen being light yellow, no petals missing, and no sign of other disease/insect damage), which indicates that these flowers were in similar developmental stage, there was no specific control to ensure that apple flowers collected had been opened for the same amount of time. In this respect, it is not clear if the recovered microbiomes are stable community structures, or if the Pseudomonas and Enterobacteriaceae dominated samples are different points in a temporal dynamic. A more detailed examination of the evolution of the microbiome over the development of the flower will better characterize the evolution of the apple flower microbiome. Finally, the recovered microbiomes were collected from compiled flowers from a single tree in order to generate sufficient amount of DNA for sequencing. In this regard, the recovered microbiomes are averaged communities for multiple flowers and not directly interacting populations, as would occur on a single stigma. Furthermore, certain factors such as soil chemistry, the fact that the trees were not randomized by location in the orchard, or microclimatic differences between tree stands, or even individual flowers, could drive alterations in the structure of the phytobiome, and the sampling and replication level of this study were not scaled to address these potential sources of variation. Taken together, these limitations in the study design limit the conclusions that can be inferred from these observations, but lend themselves to hypotheses that can be tested both in the laboratory and field. Understanding plant-microbe interactions are not only important to understand plant health and development but may offer novel ways to design effective and sustainable management practices for plant diseases.

\section{ACKNOWLEDGMENTS}

We thank R. Patel and P. Thiel for providing assistance in sample collection, and Yale Center for Genome Analysis for providing sequencing services.

\section{LITERATURE CITED}

Aleklett, K., Hart, M., and Shade, A. 2014. The microbial ecology of flowers: An emerging frontier in phyllosphere research. Botany 92:253-266.

Berg, G., Grube, M., Schloter, M., and Smalla, K. 2014. Unraveling the plant microbiome: Looking back and future perspectives. Front. Microbiol. 5: 148 .

Busby, P. E., Soman, C., Wagner, M. R., Friesen, M. L., Kremer, J., Bennett, A., Morsy, M., Eisen, J. A., Leach, J. E., and Dangl, J. L. 2017. Research priorities for harnessing plant microbiomes in sustainable agriculture. PLoS Biol. 15:e2001793.

Coleman-Derr, D., Desgarennes, D., Fonseca-Garcia, C., Gross, S., Clingenpeel, S., Woyke, T., North, G., Visel, A., Partida-Martinez, L. P., and Tringe, S. G. 2016. Plant compartment and biogeography affect microbiome composition in cultivated and native Agave species. New Phytol. 209:798-811.

de Mendiburu, F. 2017. Agricolae: Statistical Procedures for Agricultural Research. R package version. http://CRAN.R-project.org/package=agricolae

de Souza, R. S. C., Okura, V. K., Armanhi, J. S. L., Jorrín, B., Lozano, N., da Silva, M. J., González-Guerrero, M., de Araújo, L. M., Verza, N. C., Bagheri, H. C., Imperial, J., and Arruda, P. 2016. Unlocking the bacterial and fungal communities assemblages of sugarcane microbiome. Sci. Rep. 6:635-644.

Dicke, M. 2016. Plant phenotypic plasticity in the phytobiome: A volatile issue. Curr. Opin. Plant Biol. 32:17-23.

Dudareva, N., and Pichersky, E. 2000. Biochemical and molecular genetic aspects of floral scents. Plant Physiol. 122:627-634.
Elmer, W. H., Thiel, P., and Steven, B. 2017. Response of sediment bacterial communities to sudden vegetation dieback in a coastal wetland. Phytobiomes J. 1:5-13.

Fernandes, A. D., Reid, J. N., Macklaim, J. M., McMurrough, T. A., Edgell, D. R., and Gloor, G. B. 2014. Unifying the analysis of high-throughput sequencing datasets: Characterizing RNA-seq, 16S rRNA gene sequencing and selective growth experiments by compositional data analysis. Microbiome 2:15.

Ferrari, T. E., Lee, S. S., and Wallace, D. H. 1981. Biochemistry and physiology of recognition in pollen-stigma interactions. Phytopathology 71:752-755.

Fürnkranz, M., Wanek, W., Richter, A., Abell, G., Rasche, F., and Sessitsch, A. 2008. Nitrogen fixation by phyllosphere bacteria associated with higher plants and their colonizing epiphytes of a tropical lowland rainforest of Costa Rica. ISME J. 2:561-570.

Gihring, T. M., Green, S. J., and Schadt, C. W. 2012. Massively parallel rRNA gene sequencing exacerbates the potential for biased community diversity comparisons due to variable library sizes: Pyrosequencing exacerbates sample size bias. Environ. Microbiol. 14:285-290.

Gloor, G. B., and Reid, G. 2016. Compositional analysis: A valid approach to analyze microbiome high-throughput sequencing data. Can. J. Microbiol. 62:692-703.

Good, I. J. 1953. The population frequencies of species and the estimation of population parameters. Biometrika 40:237-264.

Gray, M. W., Sankoff, D., and Cedergren, R. J. 1984. On the evolutionary descent of organisms and organelles: A global phylogeny based on a highly conserved structural core in small subunit ribosomal RNA. Nucleic Acids Res. 12:5837-5852.

Hamilton, N. 2016. ggtern: An Extension to "ggplot2", for the Creation of Ternary Diagrams. R package version 2.1.5. https://cran.r-project.org/ package $=$ ggtern

Hawkes, C. V., and Connor, E. W. 2017. Translating phytobiomes from theory to practice: Ecological and evolutionary considerations. Phytobiomes J. 1:57-69.

Heitefuss, R. 2012. Fire blight, history, biology, and management. J. Phytopathol. 160:440.

Hill, T. C. J., Walsh, K. A., Harris, J. A., and Moffett, B. F. 2003. Using ecological diversity measures with bacterial communities. FEMS Microbiol. Ecol. 43:1-11.

Johnson, K. B., and Stockwell, V. O. 1998. Management of fire blight: A case study in microbial ecology. Annu. Rev. Phytopathol. 36:227-248.

Johnston-Monje, D., Lundberg, D. S., Lazarovits, G., Reis, V. M., and Raizada, M. N. 2016. Bacterial populations in juvenile maize rhizospheres originate from both seed and soil. Plant Soil 405:337-355.

Leff, J. W., and Fierer, N. 2013. Bacterial communities associated with the surfaces of fresh fruits and vegetables. PLoS One 8:e59310.

Lundberg, D. S., Yourstone, S., Mieczkowski, P., Jones, C. D., and Dangl, J. L. 2013. Practical innovations for high-throughput amplicon sequencing. Nat. Methods 10:999-1002.

Martinec, T., and Kocur, M. 1964. A taxonomic study of Erwinia amylovora (Burrill 1882) Winslow et al. 1920. Int. Bull. Bacteriol. Nomencl. Taxon. 14: 5-14.

Parks, D. H., Tyson, G. W., Hugenholtz, P., and Beiko, R. G. 2014. STAMP: Statistical analysis of taxonomic and functional profiles. Bioinformatics 30: 3123-3124.

Pusey, P. L., Rudell, D. R., Curry, E. A., and Mattheis, J. P. 2008. Characterization of stigma exudates in aqueous extracts from apple and pear flowers. HortScience 43:1471-1478.

Pusey, P. L., Stockwell, V. O., and Mazzola, M. 2009. Epiphytic bacteria and yeasts on apple blossoms and their potential as antagonists of Erwinia amylovora. Phytopathology 99:571-581.

Quast, C., Pruesse, E., Yilmaz, P., Gerken, J., Schweer, T., Yarza, P., Peplies, J., and Glockner, F. O. 2013. The SILVA ribosomal RNA gene database project: Improved data processing and web-based tools. Nucleic Acids Res. 41: D590-D596.

R Core Team. 2013. R: A language and environment for statistical computing. R Core Team, Vienna, Austria.

Rognes, T., Flouri, T., Nichols, B., Quince, C., and Mahé, F. 2016. VSEARCH: A versatile open source tool for metagenomics. PeerJ 4:e2584.

Schloss, P. D., Westcott, S. L., Ryabin, T., Hall, J. R., Hartmann, M., Hollister, E. B., Lesniewski, R. A., Oakley, B. B., Parks, D. H., Robinson, C. J., Sahl, J. W., Stres, B., Thallinger, G. G., Horn, D. J. V., and Weber, C. F. 2009. Introducing mothur: Open-source, platform-independent, community-supported software for describing and comparing microbial communities. Appl. Environ. Microbiol. 75:7537-7541. 
Shade, A., McManus, P. S., and Handelsman, J. 2013. Unexpected diversity during community succession in the apple flower microbiome. mBio. 4:e00602-12-e00602-12.

Spellerberg, I. F., and Fedor, P. J. 2003. A tribute to Claude Shannon (1916-2001) and a plea for more rigorous use of species richness, species diversity and the 'Shannon-Wiener'. Index. Glob. Ecol. Biogeogr. 12: 177-179.

Stockwell, V. O., Johnson, K. B., Sugar, D., and Loper, J. E. 2010. Control of fire blight by Pseudomonas fluorescens A506 and Pantoea vagans C9-1 applied as single strains and mixed inocula. Phytopathology 100: 1330-1339.

Stockwell, V. O., McLaughlin, R. J., Henkels, M. D., Loper, J. E., Sugar, D., and Roberts, R. G. 1999. Epiphytic colonization of pear stigmas and hypanthia by bacteria during primary bloom. Phytopathology 89:1162-1168.

Sundin, G. W., Castiblanco, L. F., Yuan, X., Zeng, Q., and Yang, C.-H. 2016. Bacterial disease management: challenges, experience, innovation and future prospects: Challenges in bacterial molecular plant pathology. Mol. Plant Pathol. 17:1506-1518.
Turner, S., Pryer, K. M., Miao, V. P. W., and Palmer, J. D. 1999. Investigating deep phylogenetic relationships among cyanobacteria and plastids by small subunit rRNA sequence analysis. J. Eukaryot. Microbiol. 46:327-338.

Turner, T. R., James, E. K., and Poole, P. S. 2013. The plant microbiome. Genome Biol. 14:209.

Vanneste, J. L. 1996. Honey bees and epiphytic bacteria to control fire blight, a bacterial disease of apple and pear. Biocontrol News Inf. 17:67N-78N.

Wang, Q., Garrity, G. M., Tiedje, J. M., and Cole, J. R. 2007. Naive Bayesian classifier for rapid assignment of rRNA sequences into the new bacterial taxonomy. Appl. Environ. Microbiol. 73:5261-5267.

Wei, T., and Simko, V. 2017. corrplot: Visualization of a Correlation Matrix. https://CRAN.R-project.org/package=corrplot

Westcott, S. L., and Schloss, P. D. 2017. OptiClust, an improved method for assigning amplicon-based sequence data to operational taxonomic units. mSphere 2:e00073-17.

Wilson, M., and Lindow, S. E. 1993. Interactions between the biological control agent Pseudomonas fluorescens A506 and Erwinia amylovora in pear blossoms. Phytopathology 83:117-123. 